\title{
Desarrollo de escenarios turísticos en las comunidades sujetas al reasentamiento por el Proyecto Hidroeléctrico El Diquís en Costa Rica
}

\author{
Development of tourist sites in communities \\ subject to resettlement due to the El Diquís \\ Hydroelectric Project in Costa Rica
}

Juan Fernando Mendoza Ledezma.

Resumen: Costa Rica se coloca como el tercer país más rico en oferta hídrica de la región centroamericana y primero en per cápita de agua. El Instituto Costarricense de Electricidad implementó el Proyecto Hidroeléctrico El Diquís (PHED) el cual se desarrolló entre los cantones de Buenos Aires, Osa y Pérez Zeledón, ubicados en la zona sur de Costa Rica. El objetivo de este estudio fue identificar las instituciones que contribuyan al mejoramiento del desarrollo de escenarios turísticos en las comunidades sujetas al reasentamiento por el PHED. Se aplicó un total de 18 entrevistas, las cuales se dividen en nueve para organizaciones e instituciones y la otra mitad para los tour operadores, la cantidad se definió de acuerdo a la disponibilidad de los actores. El estudio se realizó en tres etapas: 1) Recopilación de información secundaria, 2) Recopilación de información primaria (trabajo de campo), y 3) Triangulación y análisis de la información. Una importante conclusión es que el cantón Buenos Aires no se encuentra preparado para llevar a cabo actividades de turismo debido a la falta de capacitación de la población, además de la poca infraestructura que se requiere para ofrecer servicios adecuados de turismo.

Palabras clave: Oferta hídrica, escenarios turísticos, comuni- dades, capacitación, infraestructura.

Abstract: Costa Rica is the third richest country in water supply in Central America and first in per capita water. The Costa Rican Electricity Institute implemented the El Diquís Hydroelectric Project (PHED in Spanish), which was developed among the Cantons of Buenos Aires, Osa and Perez Zeledón, located in southern Costa Rica. The aim of this study was to identify the institutions that contribute to improving tourist site development in the communities subject to resettlement due to the PHED. A total of 18 interviews were held, divided into nine for organizations and institutions and nine for tour operators; the number was defined according to the availability of the actors. The study was conducted in three stages: 1) Compilation of secondary information, 2) Compilation of primary information (field work), and 3) Triangulation and analysis of the information. One important conclusion is that the Buenos Aires Canton is not prepared to carry out tourism activities because the population lacks training, and the infrastructure required to offer adequate tourism services is poor.

Key words: Water supply, tourist sites, communities, training, infrastructure.

(Presentado: 1 de septiembre de 2017. Aceptado: 22 de octubre de 2017)

\footnotetext{
${ }^{1}$ Doctor, Profesor Investigador Corporación Universitaria Comfacauca - Facultad de Ingenierías, Tecnología Agroambiental, Colombia. E-mail: juanfermlagmail.com
} 


\section{INTRODUCCIÓN}

El recurso hídrico "surge como el mayor conflicto geopolítico del siglo XXI, ya que se espera para el 2025 que la demanda por este recurso será un 56\% mayor al actual; no obstante lo anterior, los registros y datos históricos presagian que el planeta se encamina a una escasez muy fuerte" (Gutiérrez, 2009).

Según The Worlds Water en su informe The Biennal Report on Freshwater Resources 2002-2003, Costa Rica se coloca como el tercer país más rico en oferta hídrica de la región centroamericana, con 112,4 kilómetros cúbicos, y primero en per cápita de agua con 29.579 metros cúbicos por año por habitante (Ejecutivo, 2005). Es importante cubrir con la demanda hídrica de la zona sur del país a través de la ejecución del proyecto del Instituto Costarricense de Electricidad (ICE), pero ante todo brindando alternativas para la población del área de influencia del Proyecto $\mathrm{Hi}$ droeléctrico El Diquís (PHED).

A partir de la construcción de dicha represa hidroeléctrica (lago artificial), resulta para las comunidades una oportunidad de desarrollo de proyectos locales y fomento de actividades turísticas o vinculadas a ésta, al ser el turismo desde hace veinte años la actividad económica más importante de Costa Rica (Banco Nacional de Costa Rica, 2011). Es por ello que es importante analizar el papel que cumplen las instituciones presentes en el área del proyecto en relación a la alternativa del desarrollo del fortalecimiento del turismo, lo cual hace necesario evaluar las probabilidades de adopción de esta actividad productiva como alternativa al largo plazo (considerando que debe de darse prioridad al restablecimiento de las familias en los nuevos sitios donde serán reasentadas, considerando la sostenibilidad alimentaria en primera instancia), a través del análisis participativo en los distintos escenarios de manejo sostenible a nivel de paisaje, cultura, cosmovisión, gastronomía, tradición, historia y actividades agroproductivas, como alternativa para mejorar las condiciones de la región. Por ende, la posibilidad de turismo que resulta para las comunidades como una posible opción de desarrollo a largo plazo hace necesario considerar en el proceso de identificación, la posibilidad real de adopción de esta alternativa a través del análisis de la zona, realizando un diagnóstico participativo, incluyendo la identificación de instituciones y su contribución a las diferentes situaciones encontradas.

\section{Materiales y Métodos}

\section{Localización del estudio}

El estudio se desarrolló en la zona Sur de Costa Rica, entre el cantón de Buenos Aires, Osa y Pérez Zeledón, lugar donde el ICE está llevando a cabo el estudio de impacto ambiental para desarrollar el PHED, el cual inundará aproximadamente un total de 6.815 hectáreas. Por tal razón, la investigación se llevó a cabo en el área posible de embalse tomando en cuenta las comunidades afectadas por éste. Para motivos de ubicación se tomó como referencia el cantón de Buenos Aires, que pertenece a Puntarenas, sitio de mayor desarrollo de actividades del proyecto, ubicado geográficamente entre las coordenadas $09^{\circ} 05^{\prime} 20^{\prime}$ latitud Norte y $83^{\circ} 16^{\prime} 07^{\prime \prime}$ longitud Oeste; dado que de allí se dan los desplazamientos a las comunidades involucradas debido al futuro embalse (Figura 1).

\section{Figura 1. Área de estudio.}

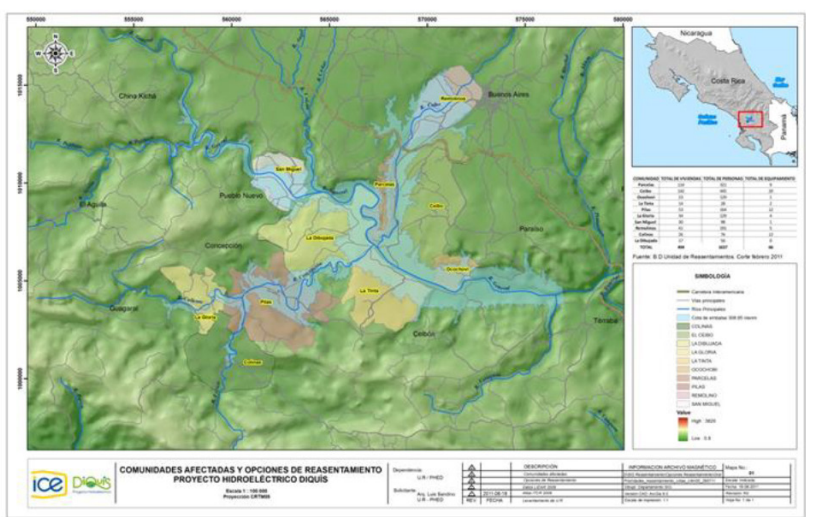

Fuente: PHED-Unidad de Reasentamientos-SIG 2011

El estudio se realizó en tres etapas divididas de la siguiente manera: 1) Recopilación de información secundaria, 2) Recopilación de información primaria (trabajo de campo), y 3) Triangulación y análisis de la información (Mendoza, 2011).

A partir de la recopilación de información tanto secundaria como primaria, se logró la identificación de actores comunitarios interesados en las actividades turísticas, los cuales en su totalidad fueron identificados a partir de las entrevistas semi-estructuradas, estudio del ICE 20092010-2011, el diagnóstico y la socialización de hallazgos; cuyo objetivo fue identificar sus intereses y perspectivas de las instituciones, asociaciones, habitantes y entidad ejecutora del proyecto, entre otras organizaciones en la toma de decisiones y la contribución al mejoramiento de 
las debilidades encontradas. Así, teniendo conocimiento de los actores que inciden en el proceso de toma de decisiones, se procedió a lograr el involucramiento de éstos, direccionado hacia el potencial de cambio de actividad tradicional productiva a la adopción del turismo como alternativa de ingreso y desarrollo. Con base a la identificación de los actores, se realizó la socialización de hallazgos en el que intervinieron las instituciones, de tal forma que se lograron construir las bases para elaborar en conjunto los lineamientos de acción como propuesta de implementación para el desarrollo del turismo por parte del trabajo que realizaran las comunidades con el apoyo del PHED, con base en una agenda en la que confluyan las diferentes visiones encaminados al objetivo común.

En el etapa de triangulación y análisis de la información, la cual es una etapa metodológica en vista al tipo de información recolectada, se buscó tomar en cuenta los diferentes puntos de vista, integrando varias apreciaciones para una mayor aproximación a la comprensión de la realidad y el posible acceso a dimensiones diferentes de la misma. De esta forma, se llevó a cabo un proceso de corroboración y correlación de información en el desarrollo de la información, es decir, desde una perspectiva plural. Esta triangulación se realizó considerando las diferentes etapas metodológicas de la investigación, partiendo de la reco- lección de datos secundarios, como la bibliografía y datos primarios, como las entrevistas, escuchando diferentes percepciones a través de la socialización de hallazgos tanto de actores afectados como afectantes, finalmente se realizó el cruce de la información para la identificación de las instituciones claves en el desarrollo del proceso de intervención. Para el análisis de la información previamente recolectada, tanto primaria como secundaria, se desarrolló un proceso de reflexión crítica proveniente de la triangulación y demás datos recolectados.

\section{Resultados Y DISCUSIÓN}

Entrevistas para organizaciones, instituciones y tour operadores

Se realizaron entrevistas a organizaciones, instituciones y tour operadores, de tal forma que se logró obtener información de actores especializados en turismo, la cual fue de interés para la investigación, en la medida que con estos datos adquiridos se logró conocer los intereses, requerimientos, estrategias y programas con los que cuentan las instituciones, principalmente en la zona sur de Costa Rica. Se aplicó un total de 18 entrevistas, las cuales se dividen en nueve para organizaciones e instituciones y la otra mitad para los tour operadores, la cantidad se definió de acuerdo a la disponibilidad de los actores (Tabla 1).

\section{Tabla 1. Listado de organizaciones, instituciones y tour operadores entrevistados}

\begin{tabular}{ll}
\hline Organización/Institución & Tour Operador \\
\hline $\begin{array}{l}\text { Cámara de Comercio, Turismo, Industria } \\
\text { y Agricultura de Pérez Zeledón }\end{array}$ & $\begin{array}{l}\text { CANTURURAL-Cámara de Turismo } \\
\text { Rural Comunitario. }\end{array}$ \\
Universidad de Costa Rica & COOPRENA TOURS-Consorcio Cooperativo \\
& $\begin{array}{l}\text { Red Ecoturística Nacional } \\
\text { Ex funcionario PNUD-Consultor en Turismo Sostenible }\end{array}$ \\
& $\begin{array}{l}\text { TURISUR-Asociación de Empresarios Turísticos del } \\
\text { Valle EI General y el Pacífico Sur de Costa Rica }\end{array}$ \\
MAG-Programa de Desarrollo Rural & $\begin{array}{l}\text { Reserva Dúrika-Antigua Cámara de Turismo } \\
\text { de Buenos Aires }\end{array}$ \\
ICT-Departamento de Desarrollo Turístico & Cámara de Turismo de Quepos \\
INA-Centro Especializado en Turismo & Cámara de Turismo de Pérez Zeledón \\
IDA & CANATUR-Cámara Nacional de Turismo \\
ACLA-P Área Conservación La Amistad-Pacífico, Buenos Aires & CANAECO-Cámara Nacional de Ecoturismo \\
UTUR-Universidad del Turismo & ACTUAR-Asociación Costarricense de \\
& Turismo Rural Comunitario
\end{tabular}


De las organizaciones entrevistadas siete cuentan con programas de turismo; solamente el Instituto de DesarroIlo Rural (IDA) y Área de Conservación La Amistad Pacífico (ACLA-P) no enfocan sus programas de trabajo en ésta área. Principalmente las organizaciones que sí cuentan con éstos se enfocan en los lineamientos del Instituto Costarricense de Turismo (ICT), establecidos en los diferentes programas de desarrollo turístico, tal como se observa en la Figura 2.

\section{Figura 2. Programas en turismo de organizaciones.}

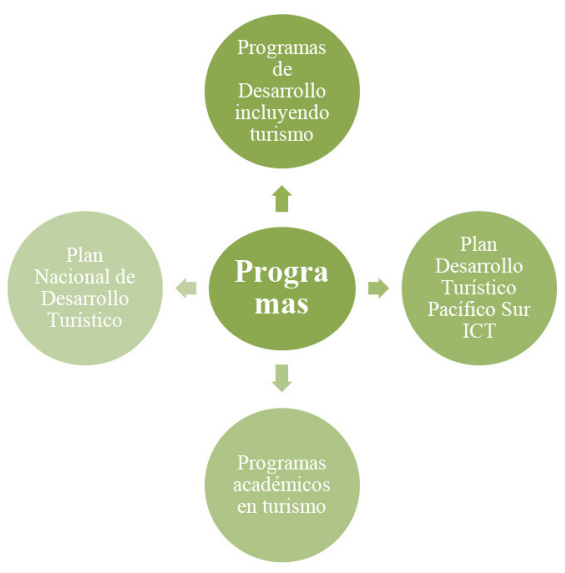

De las principales funciones que desarrollan estas instituciones, está las de la Cámara de Comercio, Turismo,
Industria y Agricultura de Pérez Zeledón, ya que representa, defiende, asesora, fortalece, capacita y desarrolla el sector turístico de dicha ciudad con proyección comunal. De esta forma, busca promocionar el turismo en la zona pacífico sur de Costa Rica, enfocados en el turismo ecológico puesto que, según la cámara, "Pacífico Sur lo tiene todo". Sumado a ello, el Ministerio de Agricultura y Ganadería (MAG) también realiza funciones en relación al turismo, ya que cuenta con un proceso de gestión territorial en la zona sur, el cual es un modelo líder tropicalizado desde el 2004 que busca construir grupos de acción territorial y asíllevar a cabo diferentes proyectos, incluyendo los de carácter turístico. La Universidad de Costa Rica (UCR) cumple un papel frente al turismo de formación de profesionales que responden al contexto social de la región, ofreciendo un programa de maestría en gestión ambiental y ecoturismo. Dentro de sus actividades se destacan los planes reguladores en los cantones de la región Brunca, Pérez Zeledón, Osa, Golfito y corredores biológicos en los que incluye actividades de desarrollo de turismo. Respecto al ICT, ha desarrollado políticas destinadas a promover el turismo sostenible e impulsar un modelo de desarrollo turístico. Para cumplir este objetivo, realiza planes de desarrollo de turismo, dentro de los cuales se encuentra el del Pacífico Sur, el cual cuenta con proyectos de turismo rural a través de alianzas con el Pacífico Central, creando un circuito turístico indígena (Tabla 2).

\section{Tabla 2. Opinión organizaciones en turismo de la zona sur Costa Rica}

\begin{tabular}{|c|c|c|c|c|c|c|}
\hline $\begin{array}{l}\text { Organización / } \\
\text { Institución }\end{array}$ & $\begin{array}{c}\text { Proyectos } \\
\text { zona sur }\end{array}$ & $\begin{array}{l}\text { Sitios para } \\
\text { visitar } \\
\text { zona sur }\end{array}$ & $\begin{array}{c}\text { B.AS } \\
\text { preparado } \\
\text { para turismo }\end{array}$ & $\begin{array}{l}\text { B.AS como } \\
\text { atractivo } \\
\text { turístico }\end{array}$ & $\begin{array}{c}\text { PHED tendrá } \\
\text { efectos positivos } \\
\text { o negativos }\end{array}$ & $\begin{array}{c}\text { Características } \\
\text { turísticas en } \\
\text { PHED }\end{array}$ \\
\hline
\end{tabular}

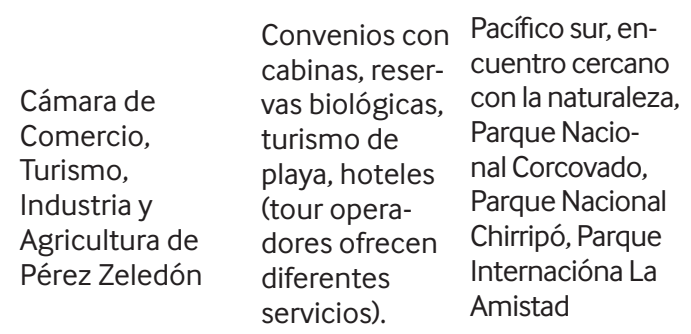

\section{Positivo: Desarrollo}

No - Por falta de

infraestructura Naturaleza Negativo: Impacto ambiental

Visitas, navegación
Universidad de No conocer Costa Rica
Visitar riqueza de la zona sur recursos naturales, ciclo vías, senderos

\begin{tabular}{|c|c|c|c|}
\hline $\begin{array}{l}\text { No - Falta } \\
\text { preparación }\end{array}$ & No conoce & $\begin{array}{l}\text { Positivos: } \\
\text { Empleo }\end{array}$ & $\begin{array}{l}\text { Senderos, visitas } \\
\text { a la represa }\end{array}$ \\
\hline
\end{tabular}




\begin{tabular}{ccccccc}
\hline $\begin{array}{c}\text { Organización / } \\
\text { Institución }\end{array}$ & $\begin{array}{c}\text { Proyectos } \\
\text { zona sur }\end{array}$ & $\begin{array}{c}\text { Sitios para } \\
\text { visitar } \\
\text { zona sur }\end{array}$ & $\begin{array}{c}\text { B.AS } \\
\text { preparado } \\
\text { para turismo }\end{array}$ & $\begin{array}{c}\text { B.AS como } \\
\text { atractivo } \\
\text { turístico }\end{array}$ & $\begin{array}{c}\text { PHED tendrá } \\
\text { efectos positivos } \\
\text { o negativos }\end{array}$ & $\begin{array}{c}\text { Características } \\
\text { turísticas en } \\
\text { PHED }\end{array}$ \\
\hline
\end{tabular}

\section{Como}

potenciales:

Propuesta de desarrollo etnoturístico, mediante el

Ex funcionario PNUD-

Especialista en turismo sostenible

\section{aprovecha-} miento de recursos actuales y potenciales dentro de los territorios indígenas (Ujarrás, Salitre, Térraba, Rey Curré y Brunca).
37 áreas protegidas de la región (24\% de total de áreas protegidas de Costa Rica)

\section{3 territorios} indígenas $(54 \%$ de los territorios del país)

)

(n)

Sí - Oferta etnoturística potencial y actual única, la cual puede ser aprovechada para desarrollar productos turísticos sostenibles novedosos e innovadores
Positivos:

Etnias, áreas protegidas, interés por desarrollo turístico

Negativos:

Falta de aprovechamiento de recursos turísticos culturales y

naturales.

Necesidad de desarrollo de visión emprendedora

Falta de planificación turística cantonal y desconocimiento sobre cómo desarrollar actividades turísticas en el territorio

\section{Positivo:}

Generación de energía

Negativo: Inundación de territorios como importancia histórica vinculada a etnias del cantón de Buenos Aires

\section{Actividades acuá- ticas y aprovecha- miento de oferta etnoturística}

MAG-Programa de Desarrollo Rural
Turismo rural comunitario en la zona sur Térraba, comuni-
dades indígenas

Si - Variedad de comunidades
Positivos:

Comunidades indígenas, vinculación al MAG con proyectos de desarrollo, capacitaciones

Negativos: Conflictos internos
Positivos:

Permite satisfacer la demanda eléctrica, empleo, oportunidades de desarrollo para los locales
Turismo rural comunitario (cluster de empresarios turísticos).

Cabalgatas, paisaje, pequeños hostales, conocer la cultura indígena

\section{ICT-}

Departamento de Desarrollo Turístico
Plan de Desarrollo Turístico pacífico sur-Turismo rural alianzas con pacífico central circuito turístico indígena

\section{INA-Centro}

Especializado en No Turismo
OSA, Costanera.

Dominical, Cerro No sabe No conoce Chirripó
No - Por ahora Positivos: Natura-

Parque la Amis- están realizando leza, turismo rural tad, OSA (Drake) los planes reguladores

Negativos: Infraestructura
No opina por desconocimiento
No opina por desconocimiento
Positivos: Beneficio para la zona con empleo

Negativo: Si no se hace de manera planificada se afecta el componente natural
Visitación, observación, atractivo cultural, turismo educativo, científico 


\begin{tabular}{ccccccc}
\hline $\begin{array}{c}\text { Organización / } \\
\text { Institución }\end{array}$ & $\begin{array}{c}\text { Proyectos } \\
\text { zona sur }\end{array}$ & $\begin{array}{c}\text { Sitios para } \\
\text { visitar } \\
\text { zona sur }\end{array}$ & $\begin{array}{c}\text { B.AS } \\
\text { preparado } \\
\text { para turismo }\end{array}$ & $\begin{array}{c}\text { B.AS como } \\
\text { atractivo } \\
\text { turístico }\end{array}$ & $\begin{array}{c}\text { PHED tendrá } \\
\text { efectos positivos } \\
\text { o negativos }\end{array}$ & $\begin{array}{c}\text { Características } \\
\text { turísticas en } \\
\text { PHED }\end{array}$ \\
\hline
\end{tabular}

Turismo rural de un grupo de señoras

IDA (restauran-
OSA (Drake) te-hotel) en el asentamiento el progreso
Negativos: Infraestructura

No, pero sí tiene potencial
Turismo rural de país sí, localmente no

Positivos: Belleza natural

SI, aunque para pocos turistas por las condiciones de infraestructura
Amistad-Pacífico, Buenos Aires
Proyectos indígenas-reserva de Ujarrás. Aunque no se otorgan los permisos para su explotación.
Ascenso al cerro

Brunca.

Cerro Camut en el parque la Amistad. Valle del Silencio de Violey en Altamira.

za natural

Negativos: Falta de infraestructura

\section{Positivos: A largo} plazo trae desarro- Turismo de llo pero antes se deben capacitar aventura

\begin{tabular}{|c|c|c|c|c|c|c|}
\hline $\begin{array}{l}\text { UTUR- } \\
\text { Universidad del } \\
\text { Turismo }\end{array}$ & No & $\begin{array}{l}\text { Cerro de la } \\
\text { muerte/giras } \\
\text { universitarias / } \\
\text { avistamiento de } \\
\text { quetzales }\end{array}$ & Sí & No conoce & No conoce & No conoce \\
\hline
\end{tabular}

Como se puede observar en la Tabla 2, del total de organizaciones entrevistadas, siete cuentan o conocen proyectos en relación al turismo y sus actividades de gestión en la zona sur de Costa Rica, principalmente, direccionados al turismo rural comunitario y el trabajo con comunidades indígenas. Dentro de los lugares que identificaron de visita para el turismo se encuentran los parques nacionales de la zona, comunidades indígenas y las playas del Pacífico Sur. Aun así, siete de las organizaciones del PHED piensan que se pueden desarrollar actividades turísticas con la construcción del PHED, ya que las dos restantes no tienen conocimiento de éste. Como por ejemplo, y según sus opiniones, actividades acuáticas, aprovechamiento de oferta etnoturística, visitas a la represa, turismo rural, turismo de aventura, turismo de investigación, cabalgatas y caminatas son algunas de las actividades que se podrían llevar a cabo en el lugar. Como observación de algunas de las organizaciones, se debe hacer énfasis en el desarrollo del turismo rural comunitario, de tal forma que no se rompa el esquema de vida actual de los habitantes de las comunidades. Además, el desarrollo turístico requiere de la creación de alianzas público-privadas y articulación de la oferta, la cual representa tan solo $5 \%$ de la oferta en Costa Rica.

En lo que se refiere a los resultados obtenidos a partir de las entrevistas realizadas a los tour operadores, se halló lo que se indica a continuación. Según se observa en la Figura 3, se puede apreciar el tipo de turismo que practican los tour operadores entrevistados, así como los servicios que éstos ofrecen en las diferentes partes de Costa Rica, Zona Sur, Zona Norte, Pacífico Central, Guanacaste, Monteverde, Sarapiquí, Caribe Sur, Norte, Valle Central, para el caso de Cooprena tours. Dominical, Palmar Sur, Osa, Platanares de Pérez Zeledón, Rivas de Pérez Zeledón, San Gerardo de Dota, Uvita, San Isidro de El General para Turisur. Mientras que Canturural tiene influencia como tour operador donde se desarrolle turismo rural comunitario a nivel de país. 
Figura 3. Tipo de turismo y servicios que ofrecen tour operadores.
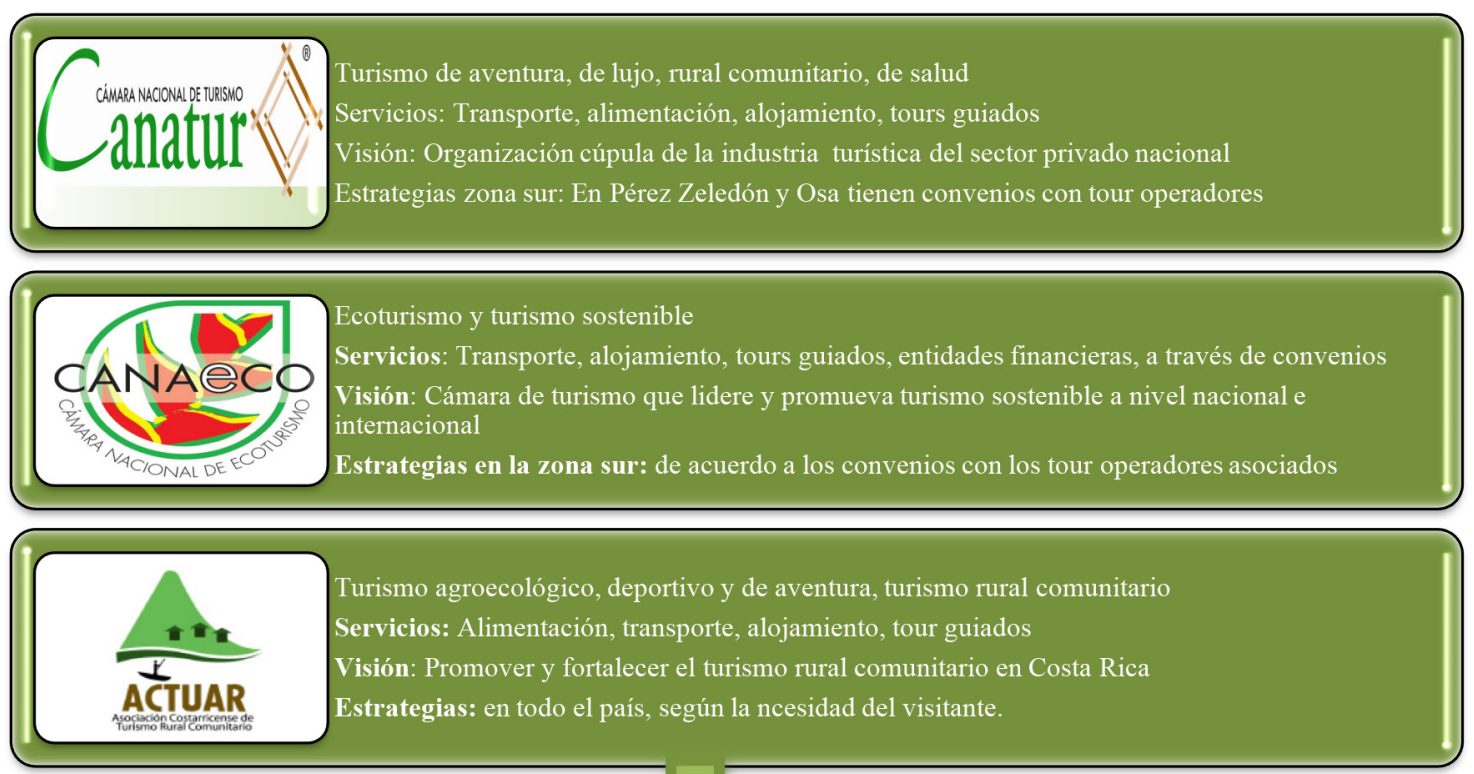
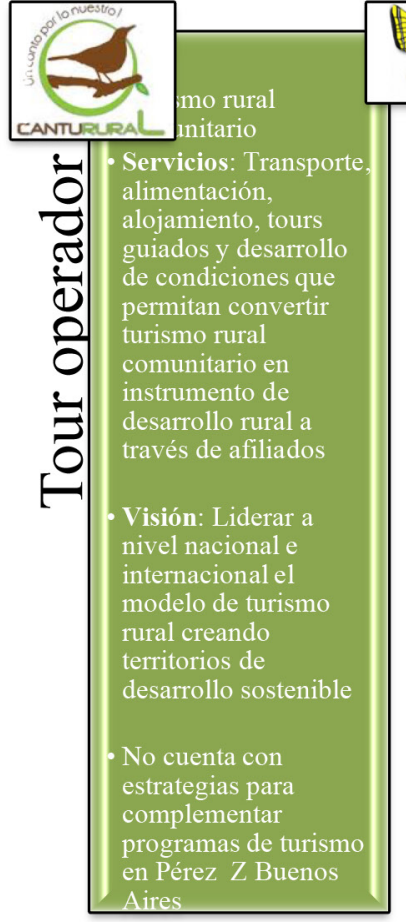

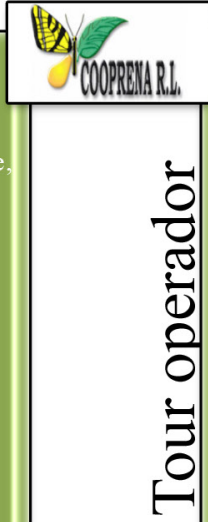

(1)

Turismo rural

comunitario y turism sostenible

Servicios:

Transporte.

alojamiento, tours

guiados, cabalgatas

cruceros.

Visión: Ser la mejor

red de organizaciones

sociales consolidada

que fortalezca el

desarrollo local a

nivel nacional e

internacional en

turismo sostenible.

Lo más cercano a

estrategias y

programas de turismo

en la zona sur es

Sierpe-Térraba

(ecoturismo) y el

Parque Nal. Chirripó.

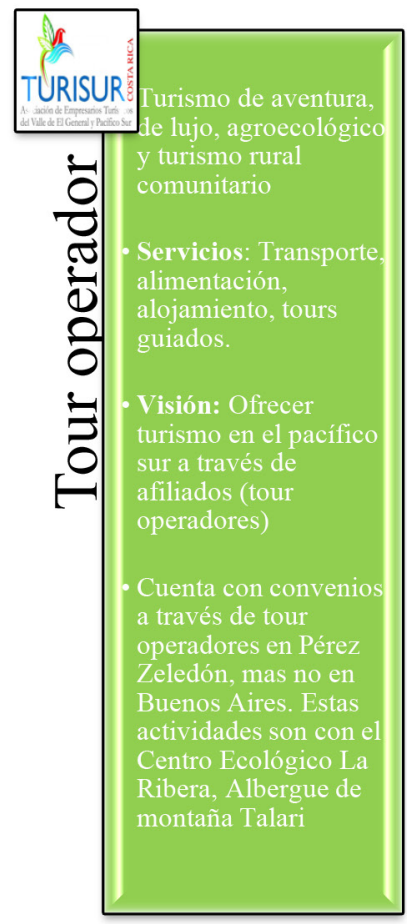


De los tour operadores entrevistados, dos consideran que Buenos Aires sí está preparado para turismo (Turisur, Canturural) por su riqueza natural y porque en la zona existen varias empresas y socios que ofrecen servicios de turismo que podrían apoyar la realización de actividades en relación a éste. En lo que se refiere a los efectos del PHED para el turismo, opinan que tendrá afectación ambiental, sin embargo traerá desarrollo, realización de estudios, conocer y observar el comportamiento del proyecto relacionado con las actividades de las comunidades aledañas; aprovechando los atractivos que puede generar la laguna, combinado con el aprovechamiento del potencial agrícola de la zona. En relación a las Cámaras de Turismo de Buenos Aires, Pérez Zeledón y Quepos, cercanas a la zona del área de investigación y de importancia turística, los resultados obtenidos se plantean en la Tabla 3.

\section{Tabla 3. Opinión cámaras de turismo sobre la zona sur Costa Rica}

\begin{tabular}{|c|c|}
\hline Cámara de turismo & Opinión \\
\hline $\begin{array}{l}\text { Reserva Dúrika } \\
\text { Buenos Aires }\end{array}$ & $\begin{array}{l}\text { Turismo rural comunitario. } \\
\text { Servicios: Transporte, alimentación, alojamiento, tours guiados } \\
\text { Visión: Trabajo comunitario en turismo. } \\
\text { Estrategias: Trabajo con estudiantes nacionales y extranjeros en reserva Dúrika. } \\
\text { Buenos Aires sí está preparado para turismo. } \\
\text { PHED traerá efectos positivos (empleo) y negativos (impacto ambiental) } \\
\text { y realizar actividades de avistamiento de aves y navegación en el embalse. }\end{array}$ \\
\hline $\begin{array}{l}\text { Cámara de Turismo } \\
\text { de Quepos }\end{array}$ & $\begin{array}{l}\text { Trabajo con tour operadores que ofrecen distintos tipos de turismo. } \\
\text { Servicios: Asociación que representa los sectores asociados al cantón. } \\
\text { Visión: Trabajo con comités de comisiones con plan de } \\
\text { mercadeo de turismo para el cantón. } \\
\text { No realiza actividades en Buenos Aires. } \\
\text { Buenos Aires no está preparado para turismo por falta de infraestructura. } \\
\text { PHED traerá desarrollo y empleo, pero también impacto ambiental. Y al ser } \\
\text { una represa institucional tendrá regulaciones para visitarla. }\end{array}$ \\
\hline $\begin{array}{l}\text { Cámara de Turismo } \\
\text { de Pérez Zeledón }\end{array}$ & $\begin{array}{l}\text { Turismo ecológico. } \\
\text { Servicios: Alojamiento, tours guiados. } \\
\text { Visión: Conservar recursos naturales, distribuir riqueza e ingresos entre comunidades. } \\
\text { Estrategia: Portal de internet para que los tour operadores ofrezcan servicios en } \\
\text { zona sur (Uvita, Dominical, Pérez Z, Puerto Jiménez, Golfito). } \\
\text { Buenos Aires no está preparado para turismo por la incipiente infraestructura } \\
\text { y baja calidad en los servicios. } \\
\text { PHED traerá efectos positivos, pero se debe capacitar a la población } \\
\text { para aprovechar el potencial como los tours por el embalse, pesca, } \\
\text { cabalgatas, avistamiento de aves y hotelería. }\end{array}$ \\
\hline
\end{tabular}

Es importante mencionar que de estas tres Cámaras de Turismo ninguna ha tenido experiencias de trabajo en relación al trabajo comunitario con el funcionamiento de hidroeléctricas. Respecto a los ingredientes faltantes a nivel de Buenos Aires para que repunte como otras regiones en temas de turismo, los entrevistados de dichas cámaras respondieron que se debe aprovechar las riquezas naturales y culturales, además del potencial agrícola; pero ante todo se deben realizar programas de capacitación, fomentar la formación profesional, conocimiento en términos económicos de tal forma que todo el conjunto de actividades a desarrollar se haga de manera organiza- 
da y previamente planificada; aprovechando el desarrollo que puede tener la zona sur con la construcción del PHED. Adicionalmente, a las comunidades se les preguntó acerca de las opciones de apoyo que pudieran tener para llevar a cabo del turismo como una opción de desarrollo; como se observa en la Figura 4 se identificaron algunas instituciones como actores clave para el apoyo a los habitantes.

\section{Figura 4. Instituciones y organizaciones de apoyo se- gún las comunidades.}

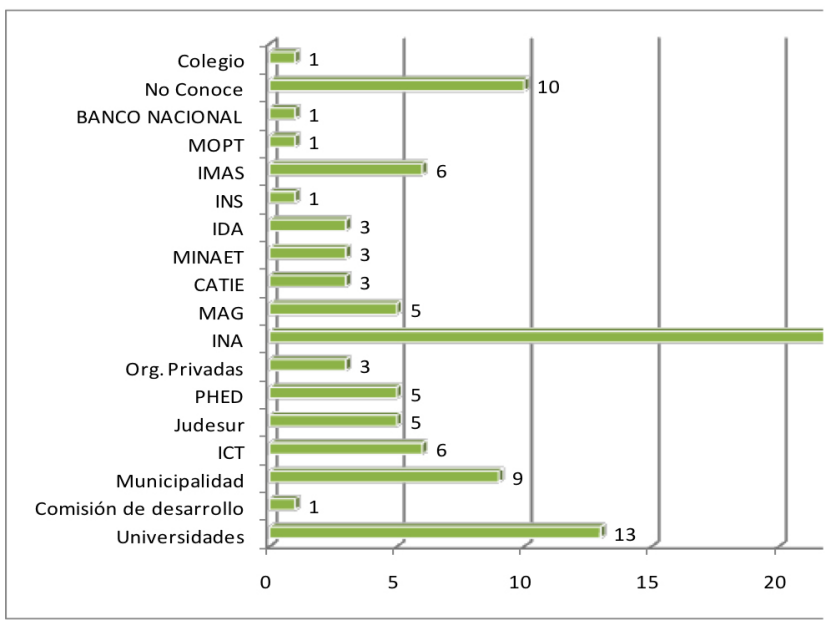

Debido a la falta de capacitación con la que cuentan las comunidades frente al desarrollo de una actividad como el turismo, la institución que identifican podría apoyar en esta temática es el INA (22\%), seguida del apoyo de las universidades (13\%), la municipalidad (9\%), el Instituto Mixto de Ayuda Social (IMAS) (6\%) y otras como el Ministerio de Agricultura y Ganadería (MAG), Junta de Desarrollo de la Zona Sur (JUDESUR ) y el PHED (5\%). En menor cantidad también mencionaron al Instituto de Desarrollo Agrario (IDA), Ministerio de Ambiente Energía y Telecomunicaciones (MINAET), Centro Agronómico Tropical de Investigación y Enseñanza (CATIE). Organizaciones privadas, Instituto Nacional de Seguros (INS), Banco Nacional (BN), Ministerio de Obras Públicas y Transportes (MOPT). Mientras que el $10 \%$ de los entrevistados dijo no conocer instituciones que podrían apoyar el desarrollo de este tipo de actividad.

\section{CONCLUSIONES}

Buenos Aires está preparado para el turismo, el $40 \%$ de las organizaciones entrevistadas respondió positivamente, justificando principalmente que el cantón cuenta con una oferta etnoturística potencial y actual única, la cual puede ser aprovechada para desarrollar productos turísticos sostenibles novedosos e innovadores, destacando también la belleza natural. Mientras que el $60 \%$ restante afirmó que Buenos Aires no se encuentra preparado para desarrollar turismo debido a la carencia en infraestructura, falta de capacitación, poca planificación turística cantonal y desconocimiento sobre cómo desarrollar actividades turísticas en el territorio.

Finalmente, las opiniones recolectadas por parte de las organizaciones frente al PHED y su relación a futuro con el desarrollo de turismo, permitió ver que se piensa que éste traerá tanto efectos positivos como negativos, ya que se va a satisfacer la demanda de energía, beneficio para la zona por la oferta de empleo y generación de desarrollo. Pero si no se realiza de manera planificada se puede ver afectado el componente natural; además de la inundación de territorios como importancia histórica vinculada a etnias del cantón de Buenos Aires que conlleva la construcción del proyecto. Las Cámaras de Turismo entrevistadas tienen conocimiento de las condiciones y dinámica del turismo de la zona sur; Buenos Aires no se encuentra preparado para llevar a cabo actividades de turismo debido a la falta de capacitación de la población, además de la poca infraestructura que se requiere para ofrecer servicios adecuados de turismo.

Es importante realizar un análisis preliminar de la demanda en cuanto a los requerimientos de organizaciones e instituciones expertas en turismo con el objetivo de verificar, por ejemplo, el potencial de la zona objeto de estudio, intereses de tour operadores, de turistas y número de visitas; además de su opinión respecto a la propuesta de turismo planteada para las comunidades sujetas al proceso de reasentamiento con base en que el proceso de investigación y la propuesta planteada. Si bien es cierto que el turismo actualmente es la principal actividad generadora de divisas en Costa Rica, comparativamente con otras actividades productivas tradicionales; también significa un aumento del costo de vida, ya que tiene un efecto de crecimiento en la economía, ocasionando según el comportamiento del mercado un aumento en el precio de bienes y servicios, que no son accesibles para algunos sectores de la población.

Existen diferentes oportunidades para desarrollar el turismo basado en la creación de la asociatividad a través de 
un enfoque sistémico con diversas organizaciones o instituciones presentes en Costa Rica las cuales ofrecen servicios tanto de capacitación y formación de capacidades como en la oferta de recursos de financiamiento para la implementación de ideas en turismo.

\section{Referencias}

Banco Nacional de Costa Rica. (2011). Banca de turismo. Consultado el 27 de septiembre de 2011. Disponible en http://www.bncr.fi.cr/bn/turismo/ index.asp

Gutiérrez, I. (s.f.). Diagnóstico rápido para la elaboración de modelo de gestión de las cuencas de los ríos banano y bananito, Limón, Costa Rica. Turrialba, Costa Rica. 83p.

Mendoza, J.F. (2011). Escenarios de alternativas de turismo en el área puntual del Proyecto Hidroeléctrico El Diquís, zona sur, Costa Rica. Tesis de Magíster. Turrialba, CR, CATIE. 209p.

Mendoza, J.F. (2015). Creation of tourist routes and guidelines of action in the communities subject to resettlement, hydroelectric the Diquis Project, as a proposal of implementation, Southern, Costa Rica. International Journal of Environmental Planning and Management 1, 3, 105-120. 